\title{
Eops: Calambres nocturnos en embarazadas
}

\author{
Nocturnal leg cramps in pregnant women
}

Victoria Wurcel

\begin{abstract}
Resumen
Se evalúa la evidencia acerca de los posibles tratamientos para los calambres en embarazadas de más de 20 semanas de edad gestacional. La mejor evidencia de eficacia disponible se relaciona a la suplementación de magnesio, ya sea en forma de lactato o citrato, a una dosis de 5 mmoles a la mañana y 10 mmoles a la tarde. La evidencia sobre la suplementación de calcio, cloruro de sodio y multivitamínicos es débil por lo que no son recomendables. Los expertos recomiendan ejercicios y masajes en las piernas, aunque carecemos de evidencia para demostrar su eficacia.
\end{abstract}

\section{Abstract}

Available evidence about treatment efficacy of pregnancy related leg cramps is revised. Best evidence of efficacy is related to supplementation of $5 \mathrm{mmols}$ of magnesium morning time and $10 \mathrm{mmols}$ evening time. Calcium, sodium chloride and multivitamin preparations are related to weaker evidence of efficacy and can not be recommended. Experts recommend exercises and leg massage, but evidence related to its efficacy is lacking.

Palabras clave: calambres nocturnos en las piernas-gestación-tratamiento-compuestos de magnesio. Key words: leg nocturnal cramps, pregnancy, treatment, magnesium compounds.

Wurcel V. Tratamiento de los calambres nocturnos en embarazadas. Evid. actual. práct. ambul; 10(2): 61-62, mar-abr.2007.

\section{Escenario}

Una médica de familia efectúa el control de salud a una embarazada sana de 29 semanas de edad gestacional. Ella la consulta por calambres en ambos miembros inferiores que le impiden conciliar el sueño. El examen físico es normal, así como un análisis de sangre que se había solicitado de "rutina". La médica decide buscar si existe algún tratamiento efectivo ya que cree recordar que "podía darse magnesio o algun polivitamínico".

\section{Pregunta}

¿En embarazadas sanas de más de 20 semanas de edad gestacional que presentan calambres de miembros inferiores (población) el magnesio y los polivitamínicos (intervención) son útiles para disminuír los síntomas (resultado)?

\section{Calambres en el embarazo}

Los calambres en las piernas son un problema frecuente en la segunda mitad del embarazo y son experimentados por la mitad de las mujeres embarazadas ${ }^{1}$, pudiendo ser particularmente molestos por la noche. Su frecuencia e intensidad tienden a aumentar hacia el final de la gestación y el mecanismo por el cual se producen durante el embarazo no está claro, aunque se sugieren como probables causas el enlentecimiento del retorno venoso y deficiencias nutricionales que provocan una acumulación a nivel muscular de ácidos como el láctico que causa contracciones involuntarias y dolorosas'. Si bien no causan daño muscular permanente pueden provocar incomodidad y deterioro de la calidad de vida, sobre todo por pérdida de horas de sueño en un período del embarazo que ya de por sí puede presentar alteraciones del mismo.

\section{Estrategia de la Búsqueda}

Se realizó una búsqueda en TRIP DATA BASE utilizando como palabras clave "leg cramps" and pregnancy. Se obtuvo una Revisión Sistemática que podía responder la pregunta.

\section{Resumen de la evidencia}

Young GL, Jewell D. Interventions for leg cramps in pregnancy (Cochrane Review). In: The Cochrane Library, Issue 1, 2006.
Oxford: Update Software.

\section{Objetivos}

Evaluar métodos para tratar los calambres en el embarazo. Fuente de Datos: registro de ensayos del Grupo Cochrane de Embarazo y Parto (Cochrane Pregnancy and Childbirth Group). Criterio de selección: ensayos clínicos aleatorizados de tratamientos para los calambres de piernas de más de dos semanas de duración en el embarazo.

Recopilación y análisis de los datos: dos revisores evaluaron la calidad de los ensayos y extrajeron los datos de forma independiente.

\section{Resultados Principales}

Se incluyeron cinco trabajos de calidad moderada que incluyeron a 352 embarazadas. El magnesio demostró ser beneficioso para disminuír la intensidad y la frecuencia de los calambres (OR: 0,18; IC95\% 0,05 a 0,6) en relación al placebo. Se detectó una tendencia beneficiosa aunque no estadísticamente significativa con los suplementos minerales (OR: 0,23 ; IC95\% 0,05 a 1,01) en relación al placebo. El único ensayo clínico que evaluó el tratamiento con calcio vs. placebo no mostró beneficio y los trabajos que compararon cloruro de sodio vs. placebo (OR: 0,54 ; IC95\% 0,23 a 1,29) y calcio vs. cloruro de sodio (OR 1,23; IC95 0,47 a 3,27) tampoco.

\section{Conclusión de los revisores}

La evidencia sobre la suplementación de calcio es débil y parece depender de un efecto placebo. Le evidencia en relación al cloruro de sodio tampoco es contundente y parece relacionarse al contenido de sodio de la dieta. Tampoco es posible recomedar multivitamínicos. La mejor evidencia de eficacia disponible se relaciona a la suplementación de magnesio, ya sea en forma de lactato o citrato, a una dosis de 5 mmoles a la mañana y 10 mmoles a la tarde.

\section{Conclusiones de la comentadora}

El embarazo es un periodo de la vida que puede traer aparejado un buen número de síntomas no demasiado placenteros, entre ellos los calambres en las piernas. Si bien las "futuras mamás" tratan en general de sobrellevarlos sin ningún medicamento "por el bien del bebé" como aconsejan abuelas y médi-

* Médica Especialista en Medicina Familiar. Servicio de Medicina Familiar y Preventiva del Hospital Italiano de Buenos Aires. 
cos, en ocasiones esto no es deseable ya que la calidad de vida de la mujer embarazada puede deteriorarse en forma significativa.

Hasta no hace mucho, realizar ensayos clínicos en embarazadas no era una practica habitual, dado que se las consideraba población vulnerable. Este concepto ha cambiado y existe consenso sobre la necesidad de realizar estudios en estas pacientes para poder medir realmente la eficacia y seguridad de los tratamientos aplicados, justamente como medida de protección para esta población.

Esta revisión es por eso bienvenida, ya que aporta evidencia sobre qué tratamiento es eficaz para los calambres en la embarazada. Destierra el uso del calcio y de los polivitaminicos, deja algo de dudas en relación al uso del cloruro de sodio (aunque aquí se plantea la duda sobre sus efectos no evaluados sobre la tensión arterial) y nos brinda como herramienta terapéutica las sales de magnesio, que ya son usadas en forma empírica por muchos médicos que atienden embarazadas. El problema de esta revisión es que no menciona dos temas importantes a la hora de indicar un tratamiento: no aclara la eficacia de tratamientos alternativos que quizá los médicos y las pacientes estaríamos más cómodos utilizando, como los ejercicios de estiramiento y los masajes (que son recomendados por los expertos) y tampoco brinda información sobre la seguridad a mediano o largo plazo de las sales de magnesio (la experiencia indica que son bastante inocuas para la madre y el feto pero la verdad es que carecemos de evidencia sólida para afirmarlo). Otros tratamientos que han demostrado cierta efectividad en pacientes no embarazadas (quinina) no son recomendables por su potencial tóxico y teratogénico.

Es interesante comentar que no se ha podido demostrar la eficacia del magnesio para tratar calambres no relacionados con el embarazo ${ }^{23}$.

\section{Recomendaciones para el consultorio}

La mejor evidencia de eficacia disponible se relaciona a la suplementación de magnesio, ya sea en forma de lactato o citrato, a una dosis de 5 mmoles a la mañana y 10 mmoles a la tarde.

Los expertos del Centro Latinoamericano de Perinatología (CLAP) y la revisión Cochrane comentada recomiendan ejercicios de estiramiento que pueden realizarse de la siguiente manera: de cara a la pared, separada de ella por $30 \mathrm{~cm}$ y con las plantas de los pies bien apoyadas en el piso, llevar el peso del cuerpo hacia adelante, manteniendo esa posición durante 20 segundos, haciendo diez repeticiones tres veces por día y masajes en las piernas.

Estas medidas parecen seguras para la madre y su hijo, sin embargo no hay hasta el momento evidencia contundente que demuestre su eficacia.

Recibido el 10/12/06 y aceptado el 12/04/06

Referencias

1. Lars O Dahle, Goran Berg, Mats Hammar, Mats Hurtig, and Lasse Larsson, The Effect of Oral Magnesium Substitution on Pregnancy-Induced Leg Cramps, American $J$ Obstetrics \& Gynecology 173(1):175-180 (July 1995)

2. Frusso R, Zárate M, Augustovski F and Rubinstein A. Magnesium for the Treatment of Nocturnal Leg Cramps. A Crossover Randomized Trial A. J Fam Pract 1999 (Nov); 48: 868-871.

3. Roffe, C., Sills, S., Crome, P. and Jones, P. Randomised, cross-over, placebo controlled trial of magnesium citrate in the treatment of chronic persistent leg cramps. Medical Science Monitor 2002; 8(5), CR326-CR330

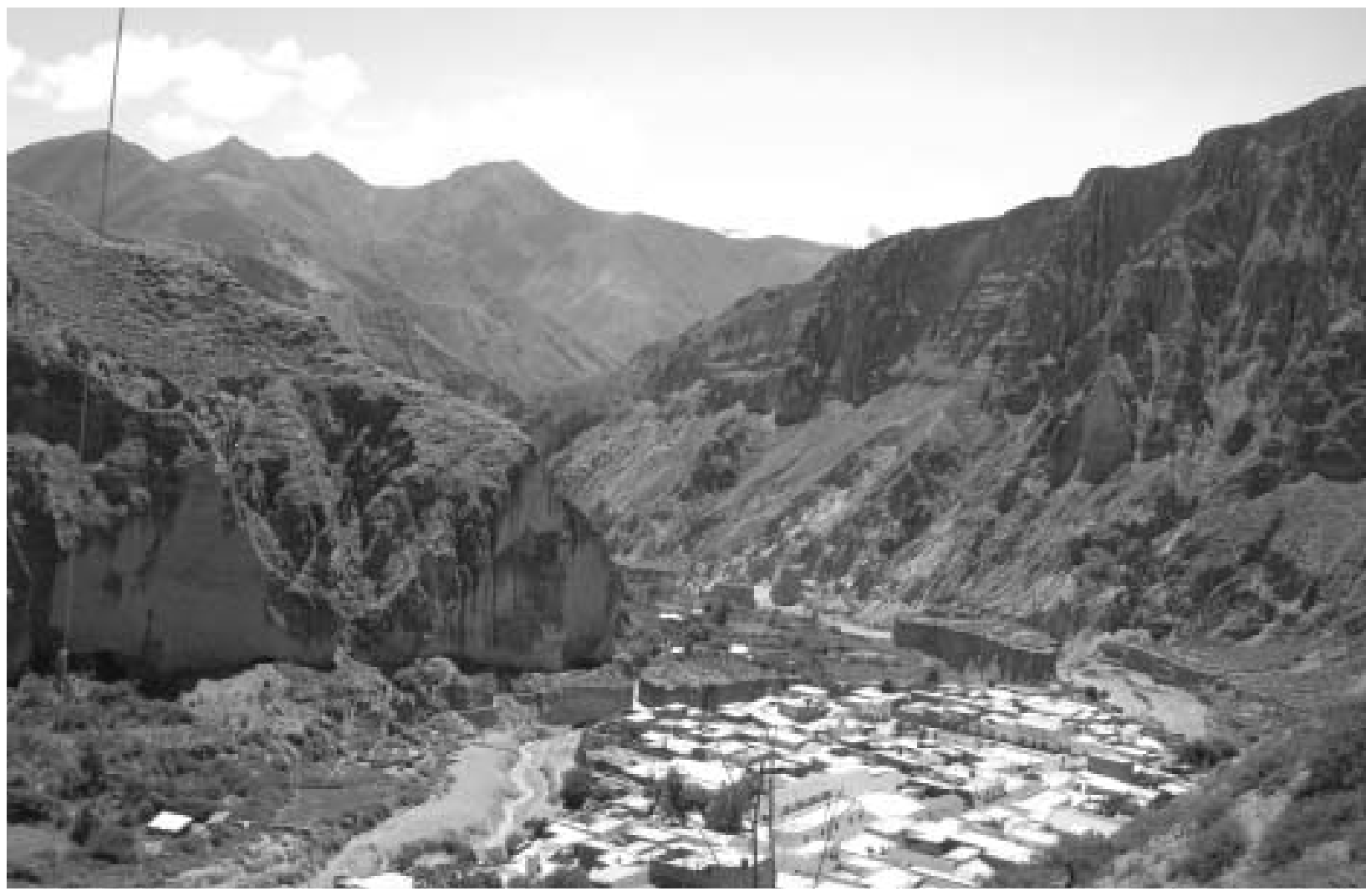

Iruya, Salta, Argentina. Cortesía: Gloria González Lázaro y Damián Terrasa. 